54, 4, pp. 1309-1318, Warsaw 2016

DOI: $10.15632 /$ jtam-pl.54.4.1309

\title{
STUDY OF A MEMS HYBRID THERMO-PZT MICRO ACTUATOR
}

\author{
Hasan Pourrostami, Navid Seyed Kazem Viliani \\ Department of Mechanical Engineering, Islamic Azad University, Abhar Branch, I.R. Iran \\ e-mail: a.pourrostami@gmail.com
}

\begin{abstract}
The current study presents an analytical model for a MEMS cantilever based hybrid thermo-piezoelectric micro actuator. The micro actuator structure consists of a unimorph type bending piezoelectric beam and a passive layer which uses the thermal bimorph principle. Primarily, under electrical and thermal loadings, the governing equations of motion are derived. After verification of the results, the deflection analysis of the micro actuator is performed. Moreover, the equivalent force at the actuator tip is obtained. To provide efficient optimization, the effects of the micro actuator geometrical and materials properties on the actuator tip deflection and force are investigated.
\end{abstract}

Keywords: hybrid micro actuator, thermo-piezoelectric, deflection, force analysis

\section{Introduction}

Micro actuators are broadly used in miniaturized systems or devices such as micro-valves and micro-switches. These actuators are designed differently from classical actuators and are mainly employed to produce mechanical motion in these devices. In order to fulfill the required performances, micro actuators need to be designed with high resolution, good accuracy, quick response time and high range of positioning capability.

Among different types of actuation mechanisms which can be classified into thermal, electrostatic, magnetic, piezoelectric and other categories, thermal and piezoelectric ones are the most prized due to their remarkable characteristics and high compatibility with MEMS devices. Some of these noticeable features in piezoelectric actuators are high positioning accuracy, rapid response time, more mechanical power. While the distinguishable feature of electrothermal actuators is a large output force with reasonable displacement.

Both piezoelectric and electrothermal micro actuators are used in the variety of applications which include micro gripper (Nguyen and Ho, 2004; Capparelli et al., 2002), scanning probe arrays (Zheng and Lu, 2004), micro positioning applications (De Cicco and Morten, 2009; Jain et al., 2005) and control valves (Roberts and $\mathrm{Li}, 2003$ ). It should be noted that the use of electrothermal micro actuators are more common. This is due to the fact that they can be easily fabricated and operated at small drive voltages. This group of actuators is ideally suited for applications that demand relatively high-output forces. However, they consume much power and show slow response time (Alwan and Aluru, 2009). On the other hand, piezoelectric actuators have the advantage of high positioning accuracy, more sensitivity and rapid response, but when compared to electrothermal micro actuators, they suffer from the limited range of deformation (Ha and Kim, 2002).

Combination of these two classes of micro actuators as a hybrid micro actuator removes their limitations and leads to modified characteristics such as low power consumption, better response time and more deflection. Rakotondrabe and Ivan (2010) attempted to integrate electrothermal and piezoelectric actuation mechanisms. They employed the thermal bimorph principle and a piezocantilever micro actuator which was made up of a piezoelectric and a passive layer. A Peltier 
module (as an external source of heat generation) was used to provide temperature variation. They used the thermal network approach to model the micro actuator. The main difference between the current research and the Rakotondrabe research is the problem-solution approach. They used the thermal network approach to model the micro actuator. In this research, the elasticity approach is used to model the micro actuator. In the elasticity approach, there is better control on designing parameters such as geometrical and material properties of the micro actuator.

Although physical modeling and temperature characterization of multi/bi layered piezoelectric cantilevers have also been studied by some researchers (Tadmor and Kósa, 2003; Ballas, 2007; Rakotondrabe et al., 2008), there is still a gap in knowledge for optimized selection of the micro actuator material and geometry based on the design requirements.

Recently, the deflection analysis of a $U$ shape hybrid thermo-piezoelectric micro actuator, in which the piezoelectric actuation mechanism was based on $d_{33}$ strain coefficient, has been performed (Pourrostami et al., 2012). In the research, the piezoelectric material properties were varied by temperature and the effects of piezoelectric material properties on the deflection of micro actuator were studied which provided efficient optimization for selecting the piezoelectric material.

The main objective of this research is the study of the effects of geometrical and material properties of the micro actuator to provide efficient optimization on its performance.

In this paper, a MEMS hybrid cantilevered thermo-piezoelectric micro actuator with $d_{31}$ actuation mechanism is developed. The micro actuator geometry is based on the presented model by Rakotondrabe and Ivan (2010). In the current study based on strength of the material, the actuator tip deflection and force are studied. Furthermore, to provide efficient optimization, the effects of the micro actuator geometrical and materials properties are investigated.

\section{Actuator modeling}

The configuration of the MEMS hybrid cantilevered thermo-piezoelectric micro actuator is illustrated in Fig. 1. This micro actuator is made of a Lead-Zirconnate-Titanate Piezoceramic (PZT) cantilever beam and a passive elastic layer (Rakotondrabe and Ivan, 2010). By applying an electrical field, the piezoelectric layer expands (or contracts) and consequently the cantilever beam bends over. Moreover, due to the existence of a difference between thermal expansions of different layers when the micro actuator is subjected to a temperature variation, the secondary bending will be induced.

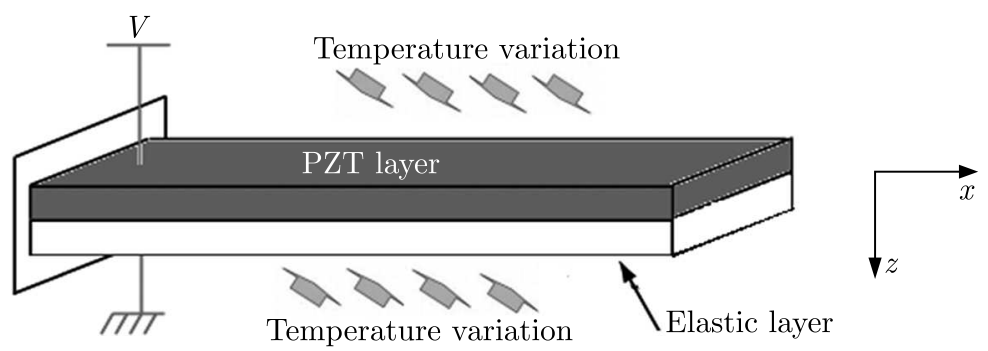

Fig. 1. The structure of the hybrid thermo-piezoelectric micro actuator (Rakotondrabe and Ivan, 2010)

Piezoelectric ceramics have very high electrical resistivity (more than $10^{9} \Omega \mathrm{m}$ ) which prevents them from any heat generation by passing electric current through the material. Therefore, in order to have thermal actuation in piezoelectric beams, an additional external heat source is needed. Based on the small size of the micro actuator, usually a uniform temperature variation on the surfaces of micro beams is considered (Rakotondrabe and Ivan, 2010). In this model, 
the classical Euler-Bernoulli beam theory (EBT) is considered, and it is assumed that the shear and electrostriction effects are negligible. Also $x$ - $z$ plane stress and $x-y$ plane strain are enforced (Devoe and Pisano, 1997).

\subsection{Constitutive relations}

For the piezoelectric materials, the general constitutive relations are (Kargarnovin et al., 2007)

$$
\boldsymbol{\varepsilon}=\mathbf{S}^{\mathrm{E}} \boldsymbol{\sigma}-\mathbf{d}^{\mathrm{T}} \mathbf{E} \quad \mathbf{F}=\mathbf{d} \boldsymbol{\sigma}-\boldsymbol{\epsilon}^{\mathrm{T}} \mathbf{E}
$$

In which $\boldsymbol{\sigma}, \boldsymbol{\varepsilon}, \mathbf{E}$ and $\mathbf{D}$ represents the columns of stress components, strain components, electrical field components and electric displacement components, respectively. Also $\mathbf{S}^{E}$, $\mathbf{d}$ and $\varepsilon$ are the compliance matrix at the constant electric field, the piezoelectric strain constant matrix and the dielectric permittivity matrix.

On the assumption of the $x-z$ plane strain which is reasonable for a wide flat micro beam, the strain component in the $y$-direction is zero. Setting $\varepsilon_{y}=0$ in equation $(2.1)_{1}, \sigma_{y}$ yields to

$$
\sigma_{y}=-\frac{1}{s_{22}}\left(s_{12} \sigma_{x}+s_{23} \sigma_{z}+d_{31} E_{z}\right)
$$

When the temperature is applied, by substituting equation (2.2) into equations (2.1), the constitutive equation reduces to

$$
\begin{array}{ll}
\varepsilon_{x}=\bar{s}_{11} \sigma_{x}+\bar{s}_{13} \sigma_{z}+\bar{d}_{31} E_{z}+\alpha \Delta T & \varepsilon_{x}=\bar{s}_{13} \sigma_{x}+\bar{s}_{33} \sigma_{z}+\bar{d}_{33} E_{z} \\
D_{z}=\bar{d}_{31} \sigma_{x}+\bar{d}_{33} \sigma_{z}+\bar{\epsilon}_{33} E_{z} &
\end{array}
$$

It should be noticed that because of small height and width of the micro actuator in comparison with its length, the thermal expansions in the $y$ and $z$ directions are neglected. Also the reference temperature is room temperature. $\bar{s}_{11}, \bar{s}_{13}, \bar{s}_{33}, \bar{d}_{31}, \bar{d}_{33}$ and $\bar{\epsilon}_{33}$ are reduced material constants of the piezoelectric for the plain stress assumed

$$
\begin{array}{lll}
\bar{s}_{11}=s_{11}-\frac{s_{12}^{2}}{s_{22}} & \bar{s}_{13}=s_{13}-\frac{s_{12} s_{23}}{s_{22}} & \bar{s}_{33}=s_{33}-\frac{s_{23}^{2}}{s_{22}} \\
\bar{d}_{31}=d_{31}-\frac{s_{12}}{s_{22}} d_{31} & \bar{d}_{33}=d_{33}-\frac{s_{23}}{s_{22}} d_{31} & \bar{\epsilon}_{33}=\epsilon_{33}-\frac{d_{31}^{2}}{s_{22}}
\end{array}
$$

Rewriting the constitutive relations in terms of stress-strain components, we get

$$
\left[\begin{array}{l}
\sigma_{x} \\
\sigma_{z}
\end{array}\right]=\left[\begin{array}{ll}
\bar{c}_{11} & \bar{c}_{13} \\
\bar{c}_{13} & \bar{c}_{55}
\end{array}\right]\left(\left[\begin{array}{l}
\varepsilon_{x} \\
\varepsilon_{z}
\end{array}\right]-\left[\begin{array}{l}
\bar{d}_{31} E_{z} \\
\bar{d}_{33} E_{z}
\end{array}\right]\right)
$$

where $\bar{c}_{11}, \bar{c}_{13}$ and $\bar{c}_{33}$ are reduced stiffness components of the piezoelectric beam. They are expressed by

$$
\bar{c}_{11}=\frac{\bar{s}_{33}}{\bar{s}_{11} \bar{s}_{33}-\bar{s}_{13}^{2}} \quad \bar{c}_{13}=-\frac{\bar{s}_{13}}{\bar{s}_{11} \bar{s}_{33}-\bar{s}_{13}^{2}} \quad \bar{c}_{33}=\frac{\bar{s}_{11}}{\bar{s}_{11} \bar{s}_{33}-\bar{s}_{13}^{2}}
$$

For a transversely isotropic piezoelectric beam with a small aspect ratio, the thickness in the $z$-direction is stress free. Therefore, it is plausible to set $\sigma_{z}=0$. By setting $\sigma_{z}=0$ in equation $(2.5), \varepsilon_{z}$ yields

$$
\varepsilon_{z}=-\frac{1}{\bar{c}_{33}}\left[\bar{c}_{13}\left(\varepsilon_{x}-\bar{d}_{31} E_{z}\right)-\bar{c}_{33} \bar{d}_{33} E_{z}\right]
$$


By substituting equation (2.7) into equations $(2.3)_{3}$ and (2.5), when the uniform temperature variation is applied, the constitutive equation reduces to

$$
\sigma_{x}^{p}=E_{p}\left(\varepsilon_{x}-\bar{d}_{31} E_{z} \alpha_{p} \Delta T\right) \quad D_{z}=\bar{d}_{31} E_{p} \varepsilon_{x}+\left(\bar{\epsilon}_{33}-\bar{d}_{31}^{2} E_{p}\right) E_{z}
$$

where $\alpha_{p}$ and $E_{p}$ are the thermal expansion coefficient and the effective elasticity modulus of the PZT layer. For the one dimensional piezoelectric beam under considered assumptions, the effective elasticity modulus is

$$
E_{p}=\bar{c}_{11}-\frac{\bar{c}_{13}^{2}}{\bar{c}_{33}}
$$

For the elastic beam under plane strain and stress assumptions by applying temperature, the constitutive relation in terms of stress-strain components is

$$
\sigma_{x}^{e}=E_{e}\left(\varepsilon_{x}-\alpha_{e} \Delta T\right)
$$

where $\alpha_{e}$ is the thermal expansion coefficient of the elastic beam and $E_{e}$ is the effective elasticity modulus of the elastic beam for the plane strain. It can be obtained from

$$
E_{e}=\frac{E}{1-\nu^{2}}
$$

\section{Deflection analysis}

The deflection of the HTP micro actuator can be derived by applying static equilibrium and strain compatibility between the layers (Devoe and Pisano 1997). Figure 2 shows a cross section of the micro actuator.
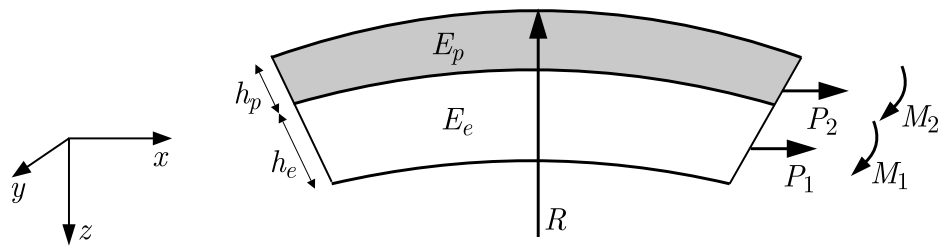

Fig. 2. The cross section of the hybrid thermo-piezoelectric micro actuator (Devoe and Pisano 1997)

In Fig. 2, $R$ represents radius of the curvature, $h_{e}$ and $h_{p}$ are heights of the elastic and piezoelectric beams. $P_{1}, P_{2}, M_{1}$ and $M_{2}$ are axial forces and moments at the cross-section of two layers. Furthermore, $E_{e}$ and $E_{p}$ are the effective elasticity modulus of the elastic and piezoelectric micro beams, respectively.

The curvature $1 / R$ of the curve $y(x)$ is expressed as follows

$$
\frac{1}{R}=\frac{d^{2} y}{d x^{2}}
$$

Then

$$
y=\frac{x^{2}}{2 R}
$$

The maximum deflection is found at the tip of the micro actuator $(x=L)$. Using the following equations, the requirements for force and moment equilibrium are satisfied (Devoe and Pisano 1997)

$$
P_{1}+P_{2}=0 \quad \frac{h_{e}}{2} P_{1}+\left(h_{e}+\frac{h_{p}}{2}\right) P_{2}+\frac{E_{e} h_{e}^{3}+E_{p} h_{p}^{3}}{12 R}=0
$$


The total strain in the elastic layer is due to axial force, bending moment and thermal effect, while the piezoelectric effect causes an additional strain component in the PZT beam. To satisfy the strain compatibility between two layers, we can write

$$
\begin{aligned}
& \varepsilon_{\text {axial }}+\varepsilon_{\text {bending }}+\varepsilon_{\text {thermal }}=\varepsilon_{\text {axial }}+\varepsilon_{\text {bending }}+\varepsilon_{\text {thermal }}+\varepsilon_{\text {piezo }} \\
& \frac{P_{1}}{E_{e} h_{e}}+\frac{h_{e}}{2 R}+\alpha_{e} \Delta T=\frac{P_{2}}{E_{p} h_{p}}-\frac{h_{p}}{2 R}+\alpha_{p} \Delta T+\frac{d_{31}}{h_{p}} V
\end{aligned}
$$

where $V, \Delta T$ and $d_{31}$ are the applied voltage, temperature variation and piezoelectric transverse strain constant, respectively.

To determine the actuator curvature, the system of equations in a matrix form is rewritten

$$
\left[\begin{array}{ccc}
1 & 1 & 0 \\
\frac{h_{e}}{2} & h_{e}+\frac{h_{p}}{2} & \frac{E_{e} h_{e}^{3}+E_{p} h_{p}^{3}}{12} \\
\frac{1}{E_{e} h_{e}} & \frac{-1}{E_{p} h_{p}} & \frac{h_{e}+h_{p}}{2}
\end{array}\right]\left[\begin{array}{c}
P_{1} \\
P_{2} \\
\frac{1}{R}
\end{array}\right]=\left[\begin{array}{c}
0 \\
0 \\
\left(\alpha_{p}-\alpha_{e}\right) \Delta T+\frac{d_{31}}{h_{p}} V
\end{array}\right]
$$

By solving equation (3.5) for the curvature $1 / R$, the actuator tip deflection using equation (3.2) is

$$
\delta=\frac{3 L^{2}\left(h_{e}+h_{p}\right) E_{e} E_{p} h_{e} h_{p}}{E_{p}^{2} h_{p}^{4}+E_{e} h_{e} E_{p} h_{p}\left(4 h_{p}^{2}+6 h_{e} h_{p}+4 h_{e}^{2}\right)+E_{e}^{2} h_{e}^{4}}\left(-\frac{\bar{d}_{31}}{h_{p}} V+\left(\alpha_{p}-\alpha_{e}\right) \Delta T\right)
$$

where $L$ is the total length of the actuator.

\section{Deflection analysis}

The equivalent generated force of the micro actuator can be obtained by the using actuator tip deflection and stiffness

$$
F=K \delta=\frac{3 E_{e q} I_{e q}}{L^{3}} \delta
$$

where $K$ is the stiffness, $E_{e q}$ and $I_{e q}$ are the equivalent elasticity modulus and moment of inertia of the two layers, respectively.

The method used to calculate the equivalent beam is based on the construction of an equivalent homogeneous section that is mechanically equivalent to the initial, heterogeneous, section (see Fig. 3). To keep flexural rigidity in the equivalent homogeneous section, the method of normalization of widths by the elasticity modulus is used. In this method, the ratio of the elastic beam width $b_{e}$ to the piezoelectric beam width $b_{p}$ should be equal to the ratio of their elasticity moduli

$$
\frac{b_{e}}{b_{p}}=\frac{E_{e}}{E_{p}}
$$

To calculate the equivalent moment of inertia, it is essential to find the location of the neutral axis of the equivalent homogeneous section $h_{e q}$

$$
h_{e q}=\frac{\sum A_{i} h_{i}}{\sum A_{i}}=\frac{h_{p} E_{p}\left(h_{e}+\frac{h_{p}}{2}\right)+\frac{1}{2} h_{e}^{2} E_{e}}{h_{p} E_{p}+h_{e} E_{e}}
$$

The equivalent moment of inertia is then expressed as follows:

$$
I_{e q}=\frac{b_{p}}{12} \frac{E_{p}^{2} h_{p}^{4}+4 h_{p} h_{e}\left(h_{p}^{2}+\frac{3}{2} h_{p} h_{e}+h_{e}^{2}\right) E_{p} E_{b}+E_{e}^{2} h_{e}^{4}}{\left(E_{e} h_{e}+E_{p} h_{p}\right) E_{p}}
$$


(a) Heterogeneous section

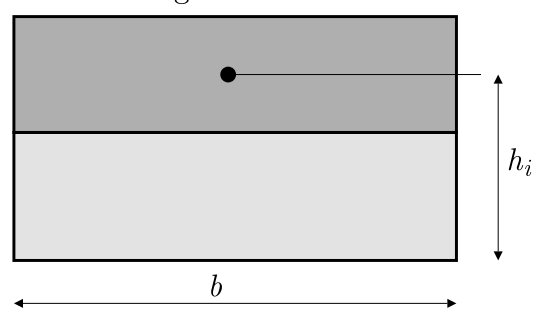

(b) Homogeneous equivalent section

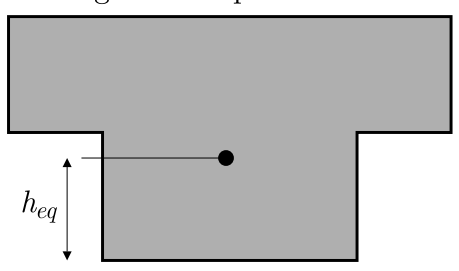

Fig. 3. (a) The cross section of the actuator with piezoelectric and elastic layers, (b) the equivalent homogenous beam section

The equivalent generated force of the micro actuator can be obtained using equations (3.6), (4.1) and (4.4)

$$
F=\frac{3}{4} \frac{3 E_{p} E_{e} h_{e} h_{p}\left(h_{e}+h_{p}\right) b}{L\left(E_{e} h_{e}+E_{p} h_{p}\right)}\left(-\frac{\bar{d}_{31}}{h_{p}} V+\left(\alpha_{p}-\alpha_{e}\right) \Delta T\right)
$$

\section{Results and discussion}

Based on the developed relations, the governing derived equations for the deflection and force of the actuator are solved numerically using the data listed in Tables 1 and 2 for geometrical and material properties, respectively. In the current study, PZT-5H and copper are used as active piezoelectric and passive elastic layers whose geometry and materials are exactly the same as the one which was presented in the experimental model by Rakotondrabe and Ivan (2010). In the first step, the validity of the obtained results is verified by comparing with the reported and experimental results (Devoe and Pisano 1997; Rakotondrabe and Ivan, 2010). Then, in order to have an optimized design, the effects of geometrical and material properties on the actuator performance are studied.

Several researches have been done on the temperature dependence of the PZT material properties which show that for PZT-5H: i) piezoelectric strain constants $d_{31}$ and $d_{33}$ increase with temperature while $d_{15}$ remains almost constant, ii) piezoelectric dielectric constants $\epsilon_{11}$ and $\epsilon_{33}$ increase with temperature, iii) for the mentioned temperature range, the elastic constants do not remarkably change with temperature (Hooker, 1998). In the current study, for verification of the results (based on the experimental results by Rakotondrabe and Ivan (2010)) the working temperature of the PZT- $5 \mathrm{H}$ is considered $50^{\circ} \mathrm{C}$. The PZT- $5 \mathrm{H}$ material properties at room and working temperature are listed in Table 2. Also it is assumed that the variation of material properties of the copper layer with temperature are negligible (Rakotondrabe and Ivan, 2010).

Table 1. Geometrical data for the actuator (Rakotondrabe and Ivan, 2010)

\begin{tabular}{|l|c|}
\hline Length of the actuator, $L[\mathrm{~mm}]$ & 15 \\
\hline Width of the actuator, $b[\mathrm{~mm}]$ & 2 \\
\hline
\end{tabular}

\subsection{Verifications}

From Eq. (3.6), it is clear that the actuator tip deflection is a linear function of the applied voltage and temperature. Substituting $\Delta T=0$ in Eq. (3.6), the hybrid micro actuator converts to the unimorph piezoelectric micro actuator (Devoe and Pisano 1997). Furthermore, based on the experimental results, two linear models have been proposed for variation of deflection versus 
Table 2. Material properties (Rakotondrabe and Ivan, 2010; Hooker, 1998)

\begin{tabular}{|l|c|c|}
\hline \multicolumn{1}{|c|}{ Title } & Symbol & Value \\
\hline \hline \multicolumn{2}{|c|}{ PZT-5H } \\
\hline Compliance matrix component $\left[\mathrm{m}^{2} / \mathrm{N}\right]$ & $s_{11}$ & $16.5 \cdot 10^{-12}$ \\
\hline Compliance matrix component $\left[\mathrm{m}^{2} / \mathrm{N}\right]$ & $s_{33}$ & $20.7 \cdot 10^{-12}$ \\
\hline Compliance matrix component $\left[\mathrm{m}^{2} / \mathrm{N}\right]$ & $s_{12}$ & $-4.78 \cdot 10^{-12}$ \\
\hline Compliance matrix component $\left[\mathrm{m}^{2} / \mathrm{N}\right]$ & $s_{13}$ & $-8.45 \cdot 10^{-12}$ \\
\hline Piezoelectric transverse strain constant $[\mathrm{V} / \mathrm{m}]$ & $d_{31}$ & $-100 \cdot 10^{-12}$ \\
\hline Expansion coefficient $\left[1 /{ }^{\circ} \mathrm{C}\right]$ & $\alpha_{p}$ & $3.6 \cdot 10^{-6}$ \\
\hline \hline \multicolumn{2}{|c|}{ Elastic layer $(\mathrm{copper})$} \\
\hline Axial elastic constant $[\mathrm{Pa}]$ & $E$ & $125 \cdot 10^{9}$ \\
\hline Poisson's ratio $[-]$ & $\nu$ & 0.35 \\
\hline Expansion coefficient $\left[1 /{ }^{\circ} \mathrm{C}\right]$ & $\alpha_{e}$ & $1.7 \cdot 10^{-6}$ \\
\hline
\end{tabular}

voltage and temperature (Rakotondrabe and Ivan, 2010). In this Section, the analytical results are compared with those from the proposed linear models.

Figure 4 ashows the variation of the actuator tip deflection versus the applied voltage when $\Delta T=0^{\circ} \mathrm{C}, h_{p}=0.2 \mathrm{~mm}$ and $h_{e}=0.1 \mathrm{~mm}$. The results follow a linear trend and the analytical result is in a good agreement with the experimental result.

In the next step, the actuator tip deflection as a function of the applied temperature is plotted (see Fig. 4b) at $V=0$ when $h_{p}=0.2 \mathrm{~mm}$ and $h_{e}=0.1 \mathrm{~mm}$, respectively. As it is seen in Fig. $4 \mathrm{~b}$, when a uniform temperature distribution is applied, the deflection variation is changed linearly.

(a)

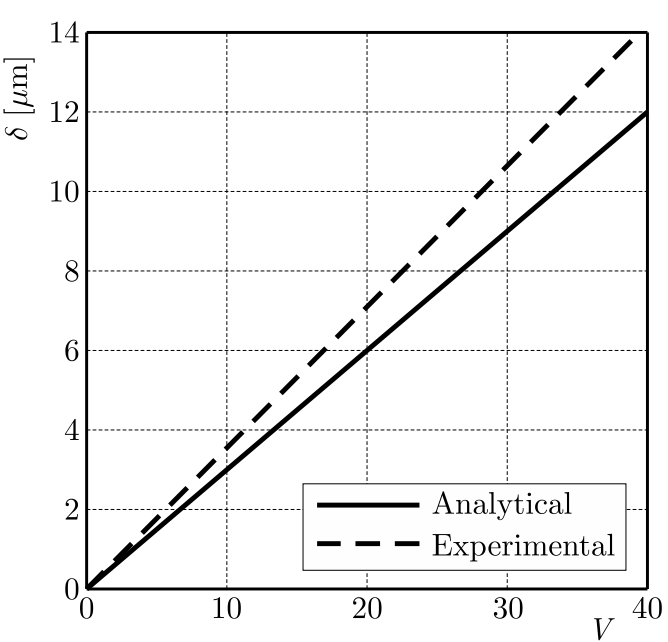

(b)

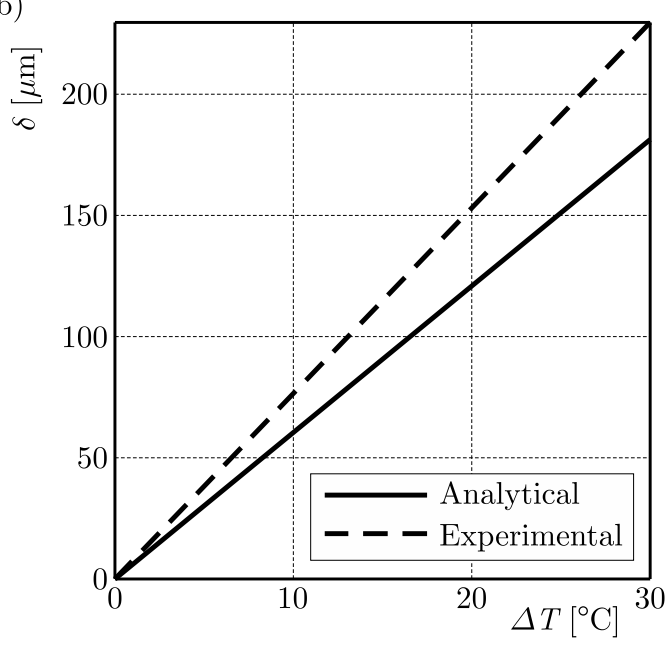

Fig. 4. The actuator tip deflection versus applied voltage (a) and uniform temperature variation (b)

The present difference between the two obtained results is due to the pyroelectric effect. The pyroelectricity is generation of an electric charge on a crystal by a change in temperature. The change in temperature slightly modifies positions of atoms within the crystal structure such that the polarization of the material changes. This polarization change gives a rise to the voltage across the crystal. As a result, the generated voltage causes greater deflection. 


\subsection{Geometry effects}

In this Section, the variations of heights of the piezoelectric and elastic layers on the actuator deflection and force are studied. Figure $5 \mathrm{a}$ illustrates the variation of the actuator tip deflection versus height of the elastic layer for two different heights of the PZT layer when $\Delta T=25^{\circ} \mathrm{C}$, $V=40 \mathrm{~V}$. Referred to this figure, smaller heights of PZT and elastic layers enable the actuator to deflect more efficiently.

Figure 5b shows the variation of the actuator force versus height of the elastic layer for two different heights of the PZT layer when $\Delta T=25^{\circ} \mathrm{C}, V=40 \mathrm{~V}$. Contrarily, greater heights of the PZT and elastic layers generate large forces in the actuator. Therefore, optimization of heights of the PZT and elastic layers can lead to the maximum efficiency of the micro actuator.

(a)

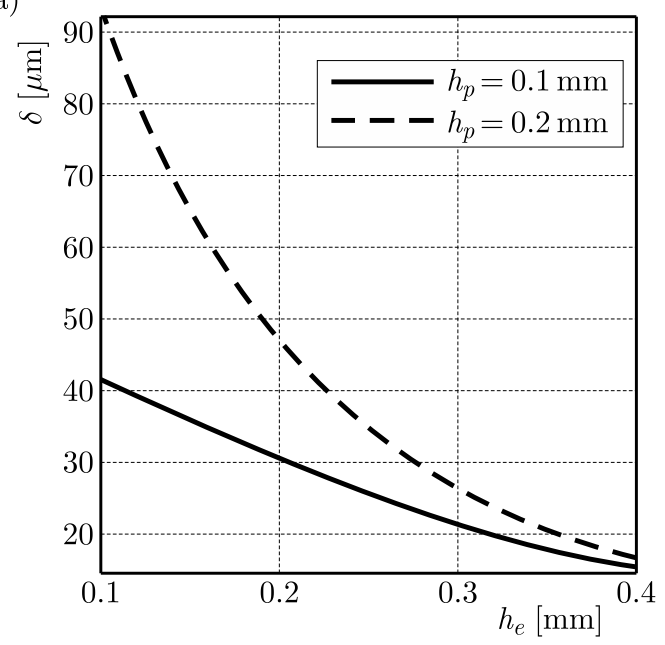

(b)

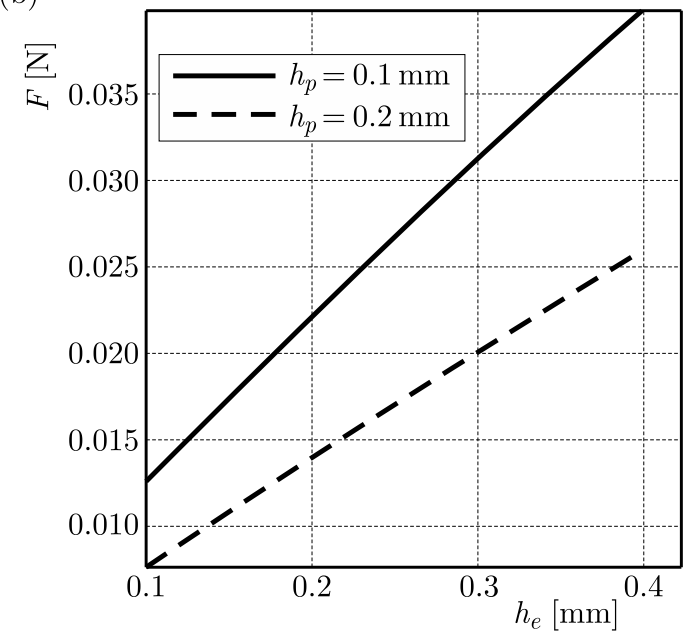

Fig. 5. (a) The actuator tip deflection versus height of the elastic layer for different heights of the PZT layer; (b) the actuator force versus height of the elastic layer for two different heights of the PZT layer

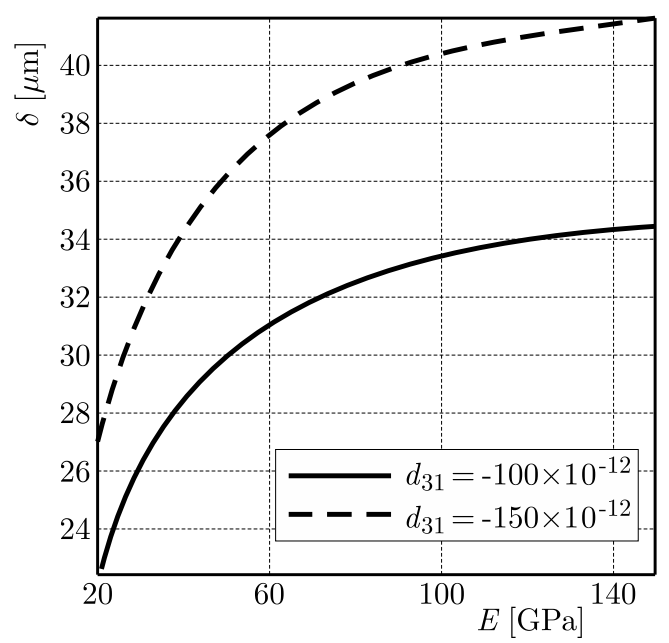

Fig. 6. The actuator tip deflection versus elasticity modulus of the elastic layer

\subsection{Material property effects}

In the present Section, the effects of the piezoelectric strain constant $d_{31}$ and the elasticity modulus of the elastic layer $E$ on the actuator deflection are investigated. The variation of actuator deflection versus elasticity modulus of the elastic layer are plotted in Fig. 6 for 
$\Delta T=25^{\circ} \mathrm{C}, V=40 \mathrm{~V}, h_{p}=0.2 \mathrm{~mm}$ and $h_{e}=0.1 \mathrm{~mm}$, respectively. There is a nonlinear variation trend when the elasticity modulus of the elastic layer increases. It means that a stiffer elastic layer leads to a greater deflection and force in the actuator. Furthermore, when the piezoelectric strain constant $d_{31}$ increases indirectly with the temperature, the actuator deflection and force rise.

\section{Conclusion}

In the present study, static analysis is performed for the proposed MEMS hybrid micro actuator which applies both the piezoelectric and thermal actuation. To sum up, the most important conclusions arising from this study are as follows:

- By increasing the applied voltage and temperature gradient, the actuator force and tip deflection linearly increase.

- The actuator tip deflection is decreased upon an increase in the heights of PZT and elastic layers.

- An indirect increase in the piezoelectric transverse strain constant $d_{31}$ by growth of the temperature give a rise to the actuator force and tip deflection.

- Making use of a stiffer elastic layer rises the nonlinear increasing trend of the actuator force and tip deflection.

- Increasing the temperature not only increases thermal expansions of the layers and, as a result, increases deflection, but also affects the piezoelectric strain constant, which enlarges deflection as well.

\section{References}

1. Alwan A., Aluru N,, 2009, Analysis of hybrid electrothermomechanical microactuators with integrated electrothermal and electrostatic actuation, Journal of Microelectromechanical Systems, 18, 1126-1136

2. Ballas R., 2007, Piezoelectric Multilayer Beam Bending Actuators, Static and Dynamic Behavior and Aspects of Sensor Integration, Springer, 1st Edit., ISBN: 978-3-540-32641-0

3. Capparelli D.J., Frecker M.I., Simpson T.W., Snyder A., 2002, Design of a PZT bimorph actuator using a metamodel-based approach, ASME Journal of Mechanical Design, 124, 354-357

4. De Cicco G., Morten B,, 2009, Thick-film piezoelectric actuators for micro positioning, Journal of Intelligent Material Systems and Structures, 20, 1689-1697

5. Devoe D., Pisano P., 1997, Modeling and optimal design of piezoelectric cantilever microactuator, Journal of Microelectromechanical Systems, 6, 3, 266-270

6. Ha S., Kim Y., 2002, Analysis of a piezoelectric multi-morph in extensional and flexural motions, Journal of Sound and Vibration, 253, 5, 1001-1014

7. Hooker M.W., 1998, Properties of PZT-Based Piezoelectric Ceramics Between -150 and $250^{\circ} \mathrm{C}$, Lockheed Martin Engineering \& Science Co., Hampton, Virginia

8. JaIn A., Qu H., Todd S., XIE H., 2005, A thermal bimorph micromirror with large bi-directional and vertical actuation, Sensors and Actuators A: Physical, 122, 9-15

9. Kargarnovin M.H., Najafizadeh M.M., Viliani N.S., 2007, Vibration control of a functionally graded material plate patched with piezoelectric actuators and sensors under a constant electric charge, Smart Materials and Structures, 16, 1252-1259

10. NGUYEN N.T., Ho S., 2004, A polymeric microgripper with integrated thermal actuators, Journal of Micromechanics and Microengineering, 14, 969-974 
11. Pourrostami H., Kargarnovin M.H., Zohoor H., 2012, Modeling and analytical solution of hybrid thermopiezoelectric micro actuator and performance study under changing of different parameters, Mechanics of Advanced Material and Structure, 22, 10, 785-793

12. Rakotondrabe M., Diouf M., Lutz P., 2008, Robust feed forward-feedback control of a hysteretic piezocantilever under thermal disturbance, IFAC World Congress, Seoul Corea, July 2008, $13725-13730$

13. Rakotondrabe M., Ivan I., 2010, Development and dynamic modeling of a new hybrid thermopiezoelectric microactuator, IEEE Transactions on Robotics, 26, 1077-1085

14. Roberts D.C., Li H., 2003, A piezoelectric microvalve for compact high frequency high-differential pressure hydraulic micropumping systems, Journal of Microelectromechanical Systems, 12, 81-92

15. TAdmor E., Kósa G., 2003, Electromechanical coupling correction for piezoelectric layered beams, Journal of Microelectromechanical Systems, 12, 899-906

16. Zheng L., Lu M., 2004, A large-displacement CMOS micromachined thermal actuator with comb electrodes for capacitive sensing, Sensors and Actuators A: Physical, 136, 2, 697-703

Manuscript received August 17, 2015; accepted for print March 7, 2016 